\title{
Body Size and the Risk of Postmenopausal Breast Cancer Subtypes in the California Teachers Study Cohort
}

\author{
Alison J. Canchola ${ }^{1}$, Hoda Anton-Culver ${ }^{2}$, Leslie Bernstein ${ }^{3}$, Christina A. Clarke ${ }^{1}$, \\ Katherine Henderson ${ }^{3}$, Huiyan $\mathbf{M a}^{3}$, Giske Ursin ${ }^{4,5,6}$, and Pamela L. Horn-Ross ${ }^{1}$ \\ ${ }^{1}$ Cancer Prevention Institute of California, Fremont, CA \\ ${ }^{2}$ Department of Epidemiology, School of Medicine, University of California, Irvine, CA \\ ${ }^{3}$ Division of Cancer Etiology, Department of Population Sciences, Beckman Research Institute, \\ City of Hope, Duarte, CA \\ ${ }^{4}$ Department of Preventive Medicine, Keck School of Medicine, University of Southern California, \\ Los Angeles, CA \\ ${ }^{5}$ Department of Nutrition, University of Oslo, Norway \\ ${ }^{6}$ Cancer Registry of Norway, Oslo, Norway
}

\begin{abstract}
Purpose-To evaluate how the association between body size and breast cancer risk varies by tumor receptor subtype, host factors and other exposures among women in the California Teacher Study cohort.
\end{abstract}

Methods-Among 52,642 postmenopausal women, 2,321 developed invasive breast cancer with known estrogen- and progesterone-receptor status (1,652 ER+PR+, 338 ER+PR-, 312 ER-PR-) between 1995 and 2007. In a subset of 35,529 with waist circumference data, 1,377 developed invasive breast cancer with known ERPR status (991 ER+PR+, 208 ER+PR-, 169 ER-PR-) between 1997 and 2007. Multivariate Cox regression was performed to estimate relative risks (RR) and 95\% confidence intervals (CI).

Results-Obesity, adult weight gain of $\geq 40$ pounds, greater abdominal adiposity and greater height increased risk of $\mathrm{ER}+\mathrm{PR}+$ breast cancer. The increased risk associated with postmenopausal obesity was limited to those who did not use hormone therapy (HT) at cohort entry (RR=1.37, 95\% CI: $1.05-1.78$ for BMI $230 \mathrm{vs.}<25 \mathrm{~kg} / \mathrm{m}^{2} ; P$-interaction=0.14) and those who were not overweight or obese at age 18 ( $P$-interaction $=0.06)$. The increased risk associated with greater abdominal adiposity was limited to those who were not also overweight or obese $(P$ interaction $=0.01$ ). Neither obesity, abdominal adiposity nor height were associated with the risk of ER-PR- tumors.

Conclusions-The effects of body size on postmenopausal breast cancer risk differed by hormone receptor subtype, and among women with ER+PR+ tumors, by HT use and early adult body size.

\section{Keywords}

breast cancer; obesity; hormone receptor status; abdominal adiposity; hormone therapy

Address correspondence to Alison Canchola, Cancer Prevention Institute of California, 2201 Walnut Avenue, Suite 300, Fremont, CA 94538; Phone: 510-608-5029; Fax: 510-608-5085; Alison.Canchola@CPIC.org.

Conflict of interest The authors declare that they have no conflict of interest. 


\section{Introduction}

There is convincing evidence that high body mass index (BMI), a measure of overall obesity, increases the risk of postmenopausal breast cancer $(1,2)$. It is likely that elevated estrogen levels, resulting from the conversion of androstenedione to estrogen in adipose tissue, as well as insulin resistance and chronic inflammation are the primary, interrelated mechanisms responsible for this association (1-3). Weight gain in adulthood is also associated with increased risk of postmenopausal breast cancer (4-6); because adult weight gain typically reflects an increase in body fat, it may have a stronger influence on risk than postmenopausal BMI (5).

Breast cancer is etiologically heterogeneous and studies have shown that risk factors vary by tumor subtype (7-9). The increase of both total and bioavailable estrogens due to obesity make it likely that BMI and adult weight gain are more strongly related to hormone receptor positive than negative breast cancers (7). However, relatively few studies have examined obesity with estrogen (ER) and progesterone receptor (PR) status jointly and results are mixed for ER-PR- tumors (4, 10-19). In addition, use of exogenous hormone therapy (HT) for relief of menopausal symptoms may modify this association further, with most studies suggesting the risk associated with BMI is limited to non-HT users or former users $(1,12$, $14,17,20-22)$.

As abdominal adipocyte dysfunction plays a role in obesity-related chronic inflammation and metabolic abnormalities (23-26), the impact of abdominal adiposity on breast cancer risk is of interest. Different measures, including waist circumference, waist-to-hip ratio, and to a lesser extent, waist-to-height ratio, have been evaluated in this regard. As with BMI and adult weight gain, abdominal adiposity has generally been associated with an increased risk of postmenopausal breast cancer (1). However, investigation of variation in risk by HT use $(20,21,27,28)$ and hormone receptor status of the tumor (13) has been limited.

We examined the effects of various body size indicators on breast cancer risk defined by joint ERPR status and potential effect modifiers including HT use, age and BMI at age 18 in the large and diverse California Teacher Study (CTS) cohort.

\section{Material and Methods}

The CTS cohort was established in 1995-96 when 133,479 active and retired female teachers, administrators and other public school professionals participating in the California State Teachers Retirement System completed an extensive questionnaire (29). This baseline questionnaire covered a wide variety of issues related to breast cancer risk and women's health, including height and weight. Two years later a follow-up questionnaire was completed which asked participants to measure the circumference of their waist and hips.

The CTS study has been approved by the Institutional Review Boards of the Cancer Prevention Institute of California, the State of California, the University of California, Irvine, the University of Southern California, and the City of Hope Medical Center.

\section{Body Composition Assessment}

On the baseline questionnaire, height (in feet and inches) and weight (in pounds) were selfreported for age 18 and at the time of completing the questionnaire. BMI for both time periods was calculated as weight (in kgs) divided by height (in $\mathrm{m}$ ) squared. Extreme BMI values, defined as BMI at baseline $<16 \mathrm{~kg} / \mathrm{m}^{2}$ or BMI at age 18 years $<15$ or $254.9 \mathrm{~kg} / \mathrm{m}^{2}$, 
were considered unreliable and those women were excluded from analysis. Weight change since age 18 (in pounds) was calculated as baseline weight minus weight at age 18 .

The follow-up questionnaire included a standard heavy-weight flexible paper tape measure (calibrated in inches on both sides with each side being a different color to prevent errors in measurement) and written, illustrated instructions on where to self-measure waist and hip circumferences. Waist was measured as the circumference one inch above the navel. Hips were measured as the largest circumference between a woman's waist and thighs. Women were instructed to measure and record each circumference twice. For each circumference (waist or hips), if the two repeated measurements were within 3 inches of each other, the average was used in the analysis. If the two repeated measurements differed by more than 3 inches or if either measurement was less than 20 inches the data were considered unreliable and the woman was excluded from the analysis. In addition, if the final waist and hip circumference values were extreme in relation to each other [i.e., waist circumference less than 26.5 inches (the $10^{\text {th }}$ percentile) and hip circumference greater than 45 inches (the $90^{\text {th }}$ percentile) or waist circumference greater than 39.5 inches (the $90^{\text {th }}$ percentile) and hip circumference less than 35.5 inches (the $10^{\text {th }}$ percentile)] the data was considered unreliable and the woman was excluded from the analysis. Waist-to-height ratio (WHtR) was calculated as waist circumference divided by height (in inches). Waist circumference measures visceral organs and subcutaneous and visceral fat while hip circumference measures muscle mass, fat mass and skeletal frame. Waist-to-hip ratio (WHR) is a measure of body fat distribution (abdominal vs. hip) but reflects both fat and muscle, whereas WHtR is considered a measure of visceral fat that is independent of height/body stature (Pearson correlation coefficient for WHtR and height of -0.15 in our study population) (30). In this analysis, waist circumference and WHtR were considered as measures of abdominal adiposity.

\section{Validation Study}

As part of the dietary validation study of the CTS (31), a trained interviewer took height, weight, and waist and hip circumference measurements on 317 study participants in early 2000. Pearson correlation coefficients were used to compare these measurements with the self-reported measures for the 313 women who provided height and weight on the baseline questionnaire (1995-96) and 251 women who provided waist and hip circumference on the follow-up questionnaire (1997-98). These validity coefficients were: $r=0.93$ for height, $\mathrm{r}=0.87$ for weight, $\mathrm{r}=0.87$ for BMI, $\mathrm{r}=0.85$ for waist circumference, $\mathrm{r}=0.87$ for hip circumference, $\mathrm{r}=0.84$ for WHtR, and $\mathrm{r}=0.65$ for WHR. Despite the several year time lapse and hence true changes in body composition, these correlations were similar to those observed in the Iowa Women's Health Study cohort, in which interviewers took measurements within 6 weeks of the self-reported measures (32).

\section{Follow-up}

The CTS cohort is followed for new cancer diagnoses, death, and changes of address. Annual linkage between the CCR and the cohort membership is used to identify incident cancer cases occurring in California residents. The CCR is a state-mandated, populationbased cancer registry covering the entire state of California. It has interstate agreements with 13 other states for case sharing purposes, is part of the National Cancer Institute's Surveillance, Epidemiology, and End Results (SEER) program and is estimated to be over 99\% complete(33). The high standards maintained by SEER and the CCR mean that followup for cancer outcomes is virtually complete as long as the cohort members reside in California. 
Linkage with California and national mortality records (the Social Security Death Master File and the National Death Index) is used to ascertain date and cause of death. Changes of address are obtained by annual mailings, responses from participants and routine record linkages with multiple sources, including the US Postal Service National Change of Address database.

\section{Study Population}

For analyses based on weight and height data, we sequentially excluded women who at baseline: were not residing in California $(n=8,867)$; had a prior $(n=6,211)$ or unknown $(n=139)$ history of breast cancer (identified by self-report or linkage with the California Cancer Registry (CCR)); were age 85 or older $(n=1,981)$; were premenopausal $(n=47,715)$ or perimenopausal $(n=2,402)$; had unknown menopausal status (i.e., were younger than age 56 and had started HT $(n=4,796)$ or had a simple hysterectomy $(n=4,281)$ before experiencing menopause, or for whom data were missing or unreliable $(n=521)$ ); or had missing or unreliable height, weight or BMI data $(n=3,406)$. Women were classified as postmenopausal at baseline if: they reported experiencing natural menopause or that their menstrual periods had stopped more than 6 months ago; both ovaries had been removed; or they were age 56 years or older at baseline and had not been classified as pre- or perimenopausal. Women who were younger than age 56 at baseline and had either started using HT or had a simple hysterectomy prior to the cessation of menses and women who had missing or inconsistent data for the variables used to define menopausal status were excluded. Among the 53,160 women eligible for this analysis, 2,839 were diagnosed with invasive breast cancer after joining the cohort and before January 1, 2008. Of these 2,839 women, 2,321 (82\%) had known ER and PR status for their tumor (1,652 ER+PR+, 338 ER +PR-, 312 ER-PR-, 19 ER-PR+). Women with invasive breast cancer whose ER or PR status was borderline or unknown $(\mathrm{n}=518)$ were excluded from all analyses; thus, 52,642 women were included in the weight and height analyses.

For analyses based on waist circumference data, we further excluded from the 53,160 eligible, sequentially, women who did not complete the follow-up questionnaire $(\mathrm{n}=12,026)$; moved out of California $(n=448)$ or were diagnosed with invasive $(n=423)$ or in-situ $(n=81)$ breast cancer between completing the baseline and follow-up questionnaire; or whose selfreported circumference data were missing or judged to be unreliable $(n=4,355)$. Among the remaining 35,827 women, 1,675 were diagnosed with invasive breast cancer after completing the follow-up questionnaire and before January 1, 2008. Of these 1,675 women, 1,377 (82\%) had known ER and PR status (991 ER+PR+, 208 ER+PR-, 169 ER-PR-, 9 ER $-\mathrm{PR}+$ ). Two hundred ninety-eight with ER or PR status borderline or unknown were excluded from all analyses; thus, 35,529 women were included in the waist circumference analyses.

\section{Data Analysis}

For height and weight analyses, follow-up time was calculated as the number of days between joining the cohort (i.e., the date the baseline questionnaire was completed) and the date of a first diagnosis of invasive $(n=2,321)$ or in-situ $(n=625)$ breast cancer, a permanent (over 4 months long) move out of California $(n=4,539)$, death $(n=6,923)$, or December 31, 2007, whichever came first. For waist circumference analyses, follow-up time was calculated as the number of days between completing the follow-up questionnaire and the first of these dates above.

Competing risk analysis was used to estimate risk of breast cancer by different hormone receptor subtypes (ER+PR+, ER+PR-, ER-PR-) (34). For analysis of a particular subtype, women diagnosed with a different subtype were censored at the time of their diagnosis, thus 
contributing person-time to the analysis until that date. Women with invasive breast cancer whose ER or PR status was borderline or unknown were excluded from all analyses. There were too few ER-PR+ cases $(n=19)$ to examine as a separate outcome. In addition, HER-2 status of tumors was not consistently available from the CCR over the time period included in this analysis and thus was not considered.

Relative risks (RR; hazard rate ratios) and 95\% confidence intervals (CI) were estimated using Cox proportional hazards regression models with age (in days) as the time-scale and stratification by age (in years) at baseline (or for waist circumference analyses, age at the completion of the follow-up questionnaire) to adjust for calendar effects. We assessed additional covariates separately for each hormone receptor subtype. Covariates were included based on prior knowledge (9) and their independent association with breast cancer risk by hormone receptor subtype in our cohort; variable definitions were chosen that best described the relationship with risk while preserving parsimony. Models predicting ER+PR+ breast cancer were adjusted for age at menarche (years from $₫$ to $\geq 17$ ), parity (parous, nulliparous) and age at first full-term pregnancy (years), history of a benign breast biopsy (yes, no), a family history of breast cancer in a first degree relative (mother or sister; yes, no), alcohol consumption in the year prior to baseline (none, $<20 \mathrm{~g} / \mathrm{d}, \geq 20 \mathrm{~g} / \mathrm{d}$ ) and use of hormone therapy at baseline (never used, past use, using estrogen-alone therapy (ET), using combined estrogen plus progesterone therapy (EPT)). Models predicting ER+PR-breast cancer were adjusted for race/ethnicity (White, non-White including Latina), parity (parous, nulliparous) and age at first full-term pregnancy (years), history of a benign breast biopsy (yes, no), a family history of breast cancer in a first degree relative (mother or sister; yes, no), alcohol consumption in the year prior to baseline (none, any) and use of HT at baseline (never used, past use, using HT). Models predicting ER-PR- breast cancer were adjusted for history of a benign breast biopsy (yes, no), a family history of breast cancer in a first degree relative (mother or sister; yes, no), average annual long-term (high school until age 54 or age at baseline if younger) moderate physical activity (hours per week) and use of HT at baseline (never used, past use, using HT) and its interaction with time-dependent age. When included as covariates, height at baseline was modeled in inches and weight at age 18 years in pounds. Analyses were repeated using a common set of covariates (including all of those described here) for all subtypes (ER+PR+, ER+PR-, ER-PR-).

We tested the assumption of proportional hazards for each covariate and main effect using a likelihood ratio test of interaction with the time-scale (continuous age) based on crossproduct terms. Covariates were coded as defined above and main effects were categorized as presented in the tables. The effect of hormone therapy use changed significantly with age for ER-PR- tumors; thus, an interaction term with time-dependent age was included as an adjustment factor. There were no violations of the proportional hazards assumption for any other covariate or for any of the main effects.

Likelihood ratio tests for trend across body measurement categories were conducted using an ordinal variable coded as the median value of each category. Likelihood ratio tests for interaction across levels of HT use at baseline (never used, past use, using ET, using EPT), age at baseline $(<65, \succ 65)$, BMI at age $18(<20,20-24,225)$ and BMI $(<25, \geq 25)$, separately, were computed based on cross-product terms with body size measures categorized as presented in the tables. To account for change over time, we repeated the obesity analysis for ER+PR+ tumors allowing both BMI and HT use (as an adjustment factor) to be updated at later questionnaires, thus making these exposures time-dependent (34). BMI was updated in 2005-06, and HT use (never used, past use, using HT) in approximately 2000-01 and 2005-06. If data were missing at a later questionnaire, the value of the time-dependent variable was set to missing until the next questionnaire or the end of follow-up. 


\section{Results}

The postmenopausal women included in this analysis had an average age of 62 years (interquartile range (IQR): 56 to 70 years) at the time they joined the cohort (Table 1). Average follow-up was 12.1 years for analyses using baseline measures and 10.1 years for the waist circumference analysis. Eighty-nine percent were Caucasian. At baseline, $21 \%$ of these women had never used HT, 13\% were past users, $27 \%$ were using ET and $29 \%$ were using EPT. The average age at baseline of HT users was lowest for EPT users (median=58 years, IQR 54-64 years) and highest for past users (median=67 years, IQR 59-74 years), with ET users and never users being intermediate (median=62 years, IQR 55-69 years and median $=65$ years, IQR 58-72 years, respectively). Twenty-nine percent of women were overweight and $16 \%$ were obese at baseline. Twenty-five percent had gained more than 40 pounds since age 18 .

Postmenopausal obesity, weight gain of $\geq 40$ pounds since age 18 , greater abdominal adiposity and greater height were associated with a higher risk of $\mathrm{ER}+\mathrm{PR}+$ tumors (Table 2). When updated data on both BMI (updated in 2005-06) and HT use (updated in 2000-01 and 2005-06) were incorporated into the analysis, the time-dependent effects for obesity (adjusted for time-dependent HT use) were essentially unchanged (RR $=1.13$, 95\% CI: 0.99 1.29 for BMI $25-29$ and $\mathrm{RR}=1.22,95 \%$ CI $1.03-1.44$ for BMI $\geq 30 \mathrm{vs}$. $<25 \mathrm{~kg} / \mathrm{m}^{2}$ ). Early adult obesity (at age 18) was associated with a reduced risk of $\mathrm{ER}+\mathrm{PR}-$ tumors $(\mathrm{RR}=0.41$, 95\% CI: $0.23-0.73$ for BMI at age $18 \geq 25$ vs. $<20 \mathrm{~kg} / \mathrm{m}^{2}$ ), while greater height increased risk. Only weight change was associated with ER-PR- tumors, with women of stable weight being at reduced risk compared to women who either gained or lost weight. Removing women with a weight loss of 10 or more pounds to focus on weight gain, continuous results did not reach statistical significance (RR=1.03, 95\% CI: $0.98-1.08$ per 10-pound increase; $P$-trend $=0.16$ across the 4 categories). Results in Table 2 were similar when adjusting all subtypes for a common set of covariates (data not shown).

We further evaluated the associations between body size and $\mathrm{ER}+\mathrm{PR}+$ tumor status to determine if they were modified by HT use, age and early adult obesity. Compared to those with BMI $<25$ and who never used HT (i.e., using a common reference group), the increased risk of $\mathrm{ER}+\mathrm{PR}+$ tumors associated with overweight and obesity were limited to those who were not using HT at baseline and were of a similar magnitude to the risk associated with ET use, with EPT use conferring somewhat greater risk than either obesity or ET use (Table 3 ). When stratified by HT use, overweight and obesity were associated with an elevation in risk among past HT users ( $\mathrm{RR}=1.51,95 \% \mathrm{CI}$ : $1.06-2.16$ for BMI $25-29$ and $\mathrm{RR}=1.50,95 \%$ CI: $0.96-2.32$ for BMI $\geq 30 \mathrm{vs.}<25 \mathrm{~kg} / \mathrm{m}^{2} ; P$-trend=0.05), but not among those using ET (RR=0.97, 95\% CI: $0.76-1.23$ for BMI 25-29 and RR=1.09, 95\% CI: $0.81-1.47$ for BMI 230; $P$-trend $=0.66$ ) or EPT (RR=1.16, 95\% CI: $0.97-1.39$ for BMI $25-29$ and $\mathrm{RR}=1.12$, 95\% CI: $0.87-1.45$ for BMI $\geq 30 ; P$-trend $=0.18)(P$-interaction $=0.43)$. Combining HT strata, the effects for overweight and obesity for never/past users (RR=1.27, 95\% CI: $1.02-1.59$ for BMI $25-29$ and RR=1.37, 95\% CI: $1.05-1.78$ for BMI $\geq 30 \mathrm{vs.}<25 \mathrm{~kg} / \mathrm{m}^{2} ; P$-trend $=0.01$ ) and those for HT users (RR=1.06, 95\% CI 0.91-1.22 for BMI 25-29 and RR=1.07, 95\% CI: $0.88-1.30$ for BMI $230 ; P$-trend $=0.40$ ) showed a similar pattern, but again differences did not reach statistical significance $(P$-interaction $=0.14)$. Similarly, adult weight gain increased risk only in women not using HT (Table 4). However, abdominal adiposity was associated with increased risk only in past HT users (P-interaction $=0.08$ ) (Table 5). While the effects of all three of these body size measures were somewhat greater in women who were older at baseline, none of the age differences were statistically significant (Table 3-5).

Interestingly, compared to women who were lean in both time periods (BMI $<20$ at age 18 and $\mathrm{BMI}<25$ at baseline), women who were overweight or obese throughout life were not at 
increased risk of $\mathrm{ER}+\mathrm{PR}+$ breast cancer $(\mathrm{RR}=0.96,95 \% \mathrm{CI}$ : $0.63-1.46$ for postmenopausal BMI 25-29 and RR=0.98, 95\% CI: 0.69-1.39 for postmenopausal BMI $\geq 30$; Table 3). The highest risk was seen in those who were lean at age 18 but obese in the postmenopausal period $(\mathrm{RR}=1.66,95 \% \mathrm{CI}$ : 1.21-2.29; P-interaction=0.06). A similar pattern was seen for weight gain itself (Table 4).

Finally, we investigated whether the effects of abdominal adiposity, as measured by WHtR, differed by overall BMI. The increased risk of ER+PR+ tumors with WHtR $\searrow .50$ was limited to women who were not overweight or obese (Table 5). Compared to lean women with a WHtR $<0.50$, excess abdominal fat without overall overweight/obesity increased risk by $26 \%$ whereas overall overweight/obesity without an abdominal excess increased risk by $51 \%$. Among overweight/obese women, abdominal adiposity did not further increase risk $(\mathrm{RR}=1.22,95 \% \mathrm{CI}: 1.03-1.43)$. When stratified by BMI, greater abdominal adiposity increased risk among women with a BMI $<25$ (RR=1.30, 95\% CI: $1.07-1.58$ for WHtR $\searrow 0.50$ vs. $<0.50$ ), whereas there was no increase in risk among women who were overweight or obese (RR=0.80, 95\% CI: 0.60-1.06; $P$-interaction=0.01). A similar pattern was seen for absolute waist circumference ( $R R=1.35,95 \%$ CI: $1.11-1.65$ for waist $\geq 30$ vs. $<30$ among those with $\mathrm{BMI}<25 ; \mathrm{RR}=0.88,95 \% \mathrm{CI}$ : 0.50-1.54 among those with BMI 25 ), although the interaction was not statistically significant $(P$-interaction $=0.21)$.

\section{Discussion}

Results from our prospective cohort of California teachers show that postmenopausal obesity, adult weight gain of $\geq 40$ pounds, greater abdominal adiposity and greater height increased risk of $\mathrm{ER}+\mathrm{PR}+$ breast cancer. The increased risk associated with obesity and weight gain was limited to those who were not using HT at baseline. In addition, neither of these factors increased risk among the small proportion of women who were overweight or obese early in adult life. Finally, we observed no additional risk associated with both overall and abdominal adiposity compared to either alone. Postmenopausal obesity, abdominal adiposity, and height were not associated with ER-PR- tumors, while weight gain showed some association with increased risk.

Interestingly, adult weight gain increased risk of both the most common subtype of breast cancer, ER+PR+, and also the subtype with the worst prognosis, ER-PR-. For ER+PR+, there was a clear linear trend as risk increased with greater weight gain. Whereas for ER-PR - , any weight gain appeared to increase risk, even at lower levels, although the number of cases was small.

Five cohort studies have observed an increased risk of ER+PR+ breast cancer in postmenopausal women with greater obesity and/or weight gain $(4,12,16,17,35)$ and a sixth found elevated but not statistically significant risks (36). Findings for the associations between obesity and weight gain with ER-PR- and ER+PR- tumors have been somewhat mixed, with most cohort studies finding no statistically significant association $(4,12,16,17$, $35,36)$ or a reduced risk $(16,35)$. A meta-analysis of body weight (based on BMI or weight gain if BMI was not available) in postmenopausal women which included four of these cohort studies $(12,17,35,36)$ found an increased risk for $\mathrm{ER}+\mathrm{PR}+$ tumors (risk estimate $(\mathrm{RE})=1.74,95 \% \mathrm{CI}: 1.34-2.25$ comparing the highest vs. reference categories), no association for $\mathrm{ER}-\mathrm{PR}-$ tumors ( $\mathrm{RE}=0.90,95 \% \mathrm{CI}$ : $0.53-1.52$ ) and a decreased risk for ER $+\mathrm{PR}$ - tumors ( $\mathrm{RE}=0.64,95 \%$ CI: 0.42-0.97; including data from two of the cohorts); although the analyses did not exclude HT users in two of the four cohorts, thus, potentially reducing the estimated effect for $\mathrm{ER}+\mathrm{PR}+$ tumors (37). A meta-analysis of adult weight gain in postmenopausal women who never used HT which included three of these cohort studies $(4,12,36)$ found an increased risk for $\mathrm{ER}+\mathrm{PR}+$ tumors $(\mathrm{RE}=2.17$, 95\% CI: 1.48-2.85 
comparing the highest vs. lowest quantiles) and no association for ER-PR- tumors ( $\mathrm{RE}=1.23$, 95\% CI: 0.57-1.90) (38). In the Swedish Mammography Cohort, which looked at obesity by HT use, the positive association between obesity and $\mathrm{ER}+\mathrm{PR}+$ tumors was limited to those who never used HT (RR=1.90, 95\% CI: $1.38-2.61$ for BMI $\geq 30$ vs. <25), with no association in those who ever used HT (RR=1.18, 95\% CI: 0.78-1.81) (17). Consistent with these prospective cohort studies and meta-analyses, we found obesity and adult weight gain increased risk of $\mathrm{ER}+\mathrm{PR}+$ breast cancer, limited to those who were not using HT; were not associated with ER+PR- tumors; and obesity was not associated with ER-PR- tumors. However, we observed an increased risk of ER-PR- tumors with adult weight gain or loss. The American Cancer Society's Cancer Prevention Study II Nutrition Cohort also found elevated risk of ER-PR- tumors associated with adult weight gain ( $R R=1.78,95 \%$ CI: $0.98-3.23$ for adult weight gain of $\succeq 61$ pounds vs. a weight change of -5 to +20 pounds) (12); although this result did not reach statistical significance and the study did not evaluate weight loss of more than 5 pounds. Also, similar to our results, the National Institutes of Health-American Association for Retired Persons Diet and Health Cohort (NIH-AARP) found a weight loss of more than $2 \mathrm{~kg}$ associated with an increased risk of ER-PR- tumors ( $\mathrm{RR}=2.01,95 \%$ CI: 0.75-5.36 compared to a stable weight of -2.0 to $+9.9 \mathrm{~kg}$ change) (4). Weight gain of 10-19.9 kg in that cohort was associated with an increased risk but greater weight gain was not. The U-shaped risk curve we observed associated with weight change and the development of ER-PR- tumors is puzzling. However, the similar results observed by the NIH-AARP cohort suggest further investigation is needed, perhaps through additional pooled analyses in order to provide sufficient statistical power. Taken together, these findings show the detrimental effect of adult obesity and weight gain on breast cancer risk.

Our finding that being overweight/obese in later life does not increase a woman's risk of ER $+\mathrm{PR}+$ breast cancer if she was also overweight/obese early in adult life is consistent with results from the NIH-AARP cohort (4). Among HT non-users in that study, those who were consistently overweight or obese at ages $18,35,50$ years and study baseline were not at increased risk of overall breast cancer ( $R R=1.07,95 \% \mathrm{CI}$ : $0.77-1.47)$ when compared to those who were consistently normal weight. Similarly, in the Nurses' Health Study, while the cumulative incidence of overall breast cancer among HT non-users was increased in those with above-average weight gain ( $R R=1.19,95 \% \mathrm{CI}$ : $1.08-1.31)$, it was not elevated in women who were consistently obese at ages $18,50,60$ and 70 ( $R R=1.06,95 \%$ CI: $0.95-$ 1.17), both compared to the 'average' woman, defined as someone who gained 19 pounds between ages 18 to 50 years (39). The implication of these findings for more recent generations, however, is unclear as the relative contributions of heredity, overeating and sedentary behavior to early life obesity may differ in older vs. younger cohorts.

Our finding that neither early life nor lifetime obesity increased the risk of ER+PR+ breast cancer points to adult weight gain as the primary, but modifiable, body size risk factor. Greater height was also associated with risk, suggesting genetic as well as early life environmental, hormonal and nutritional factors may play a role $(1,40)$. The magnitude of the relative risks for both these factors was not large, i.e., on the order of $35 \%$ in the highest risk groups; however, the prevalence of adult weight gain makes this of public health importance.

Few studies have examined the effects of abdominal adiposity on breast cancer by hormone receptor status. The Iowa Women's Health Study (IWHS) cohort found a waist-to-hip ratio (WHR) $\searrow 0.90$ increased risk of postmenopausal ER+PR+ tumors (RR=1.33, 95\% CI: $1.05-$ 1.70 vs. $<0.90$ ), but was not associated with ER+PR- or ER-PR- tumors (35). Whereas the Shanghai Breast Cancer Study, a case-control study, found a greater WHR increased risk of both postmenopausal ER+PR+ and ER-PR- breast cancer (odds ratio $(\mathrm{OR})=1.73,95 \% \mathrm{CI}$ : 
1.21-2.48 for WHR $\searrow 0.843$ vs. $<0.772$ for $E R+P R+$; OR=2.92, 95\% CI: $1.78-4.79$ for ERPR-) (41). In addition, a case-control study at the National Cancer Institute in Brazil found that waist circumference and WHR were not related to hormone receptor status in a population of predominantly overweight or obese postmenopausal women (42). Our finding that the risk of $\mathrm{ER}+\mathrm{PR}+$ tumors associated with a greater waist-to-height ratio, a measure more directly reflecting visceral fat independent of stature than WHR or waist circumference (30), was limited to women who were not overweight/obese is consistent with these studies.

A potential limitation of our study is the possibility of error in self-reported anthropometric measurements. Such error could be the result of lack of knowledge, the desire to report a socially more normative value or measurement error (for waist and hip circumferences). However, to improve the accuracy of measured waist and hip circumferences, participants were provided with specific written and pictorial instructions and a standard tape measure and were asked to take and record their measurements twice. In addition, the prospective study design eliminated recall bias and, thus, if measurement error occurred in the selfreports, it was unlikely to differ systematically between cases and non-cases. Also, socially desirable responses would have been likely to attenuate elevated risk estimates rather than inflate them. Finally, in an ancillary validation study conducted within the cohort, comparison of the self-reported measures, including weight, height, circumferences and indices derived from these measures, to measurements taken by trained interviewers suggested excellent validity (see Methods for specific Pearson correlation coefficients).

A further potential limitation was that hormone receptor status was obtained from the cancer registry, which obtains reports from different pathology laboratories throughout the state. These laboratories may use different assays and/or cut points to define a positive receptor status. A study which compared SEER results to those of a centralized laboratory found substantial agreement for $\mathrm{ER}+\mathrm{PR}+$ and $\mathrm{ER}-\mathrm{PR}$ - tumors (kappa $=0.62$ for $\mathrm{ER}+\mathrm{PR}+$; kappa $=0.69$ for $E R-P R-)$ but poor agreement for $E R+P R-(k a p p a=0.30)(43)$, suggesting results are reliable for the more consistent subtypes. However, even if differences between laboratories occurred, they are unlikely to differ systematically by risk factors or body size. An additional limitation was that $18 \%$ of cases had hormone receptor status missing or borderline, due to less reporting of ERPR in earlier diagnostic years, and thus were excluded from analysis. We compared breast cancer risk factors for women with $(\mathrm{n}=2,321)$ and without $(\mathrm{n}=518$ ) hormone receptor status and found that those with a known status were slightly younger (median age at baseline 63 years, IQR 57 to 69 years compared to median age 65 years, IQR 58-71 years for women with missing or borderline ERPR status) and somewhat more likely to drink alcohol ( $70 \%$ vs. $65 \%$, respectively). There were no differences in any of the body size factors or HT use, thus, it is unlikely that excluding women with missing hormone receptor status would have biased the observed results.

A final limitation of the study was the small number of cases with ER+PR- and ER-PRtumors and hence limited statistical power to detect associations for these breast cancer subtypes. However, since different hormone receptor subtypes may have different etiologies, making these distinctions is important. In addition, our study included cancer follow-up through the end of 2007; however, among women in the CTS cohort who reported using EPT and ET in 2000-2001, 75\% and 56\%, respectively, stopped use by 2005-2006, presumably largely related to the media attention surrounding the publication of the results of the Women's Health Initiative trial (44). Given that it is likely that current HT use confers the greatest risk of breast cancer, those women who ceased using HT during the course of follow-up modified their risk. Thus the HT use categorization we used, which was based on baseline data and thereby did not capture quitting, may have resulted in slightly attenuated risks for HT users. We also did not capture women who may have started using HT around 
the time of their breast cancer diagnosis, although it is likely this number was small. However, when we examined BMI and risk of $\mathrm{ER}+\mathrm{PR}+$ tumors with HT data that was updated over time, our results were similar. Furthermore, as with any observational study, results could be due to chance or residual confounding.

Strengths of our study include the prospective design which eliminates recall bias, detailed risk factor information and virtually complete case ascertainment which minimizes selection bias due to loss of follow-up. In addition, results from our validation study suggest that error in self-reported body measures is small.

In conclusion, our findings show that greater body size, including overall obesity, weight gain, abdominal adiposity and height in postmenopausal women increased risk of ER+PR+ breast cancer but that these overall associations are likely to be modified by HT use. Among those who were overweight or obese early in adulthood, continued overweight/obesity did not increase risk, a finding that deserves additional investigation. Weight gain was associated with ER-PR- tumors, while overall obesity and abdominal adiposity were not. Thus, our findings support the importance of weight control for breast cancer prevention.

\section{Acknowledgments}

This research was supported by grant R01 CA77398 from the National Cancer Institute and contract 97-10500 from the California Breast Cancer Research Fund. The funding sources did not contribute to the design or conduct of the study, nor to the writing or submission of this manuscript. The collection of cancer incidence data used in this study was supported by the California Department of Health Services as part of the statewide cancer reporting program mandated by California Health and Safety Code Section 103885; the National Cancer Institute's Surveillance, Epidemiology and End Results Program under contract HHSN261201000036C awarded to the Cancer Prevention Institute of California, contract HHSN261201000035C awarded to the University of Southern California, and contract HHSN261201000034C awarded to the Public Health Institute; and the Centers for Disease Control and Prevention's National Program of Cancer Registries, under agreement \# 1U58 DP000807-01 awarded to the Public Health Institute. The ideas and opinions expressed herein are those of the authors and endorsement by the State of California, Department of Health Services, the National Cancer Institute, and the Centers for Disease Control and Prevention or their contractors and subcontractors is not intended nor should be inferred.

The authors would like to thank Jane Sullivan-Halley for substantial technical assistance with quality control work on the height and weight variables and the CTS Steering Committee who are responsible for the formation and maintenance of the cohort within which this study was conducted but who are not included as authors on the current paper: Ellen T. Chang, Dennis Deapen, James Lacey, Jr, David Nelson, Susan Neuhausen, Peggy Reynolds, Frederick Schumacher, Sophia Wang, and Argyrios Ziogas.

\section{Abbreviations}

$\begin{array}{ll}\text { BMI } & \text { body mass index } \\ \text { CTS } & \text { California Teachers Study } \\ \text { EPT } & \text { combined estrogen plus progesterone therapy } \\ \text { ER } & \text { estrogen receptor } \\ \text { ET } & \text { estrogen-only therapy } \\ \text { HT } & \text { hormone therapy } \\ \text { PR } & \text { progesterone receptor }\end{array}$

\section{REFERENCES}

1. Food, Nutrition, Physical Activity and the Prevention of Cancer: A Global Perspective. AICR; Washington, DC: 2007. World Cancer Research Fund and the American Institute for Cancer Rearch. 
2. Calle EE, Kaaks R. Overweight, obesity and cancer: epidemiological evidence and proposed mechanisms. Nat Rev Cancer. 2004; 4:579-91. [PubMed: 15286738]

3. Key T, Appleby P, Barnes I, Reeves G. Endogenous sex hormones and breast cancer in postmenopausal women: reanalysis of nine prospective studies. J Natl Cancer Inst. 2002; 94:60616. [PubMed: 11959894]

4. Ahn J, Schatzkin A, Lacey JV Jr. et al. Adiposity, adult weight change, and postmenopausal breast cancer risk. Arch Intern Med. 2007; 167:2091-102. [PubMed: 17954804]

5. Ballard-Barbash R, Schatzkin A, Carter CL. Body fat distribution and breast cancer in the Framingham Study. J Natl Cancer Inst. 1990; 82:286-90. [PubMed: 2299677]

6. Feigelson HS, Jonas CR, Teras LR, Thun MJ, Calle EE. Weight gain, body mass index, hormone replacement therapy, and postmenopausal breast cancer in a large prospective study. Cancer Epidemiol Biomarkers Prev. 2004; 13:220-4. [PubMed: 14973094]

7. Althuis MD, Fergenbaum JH, Garcia-Closas M, Brinton LA, Madigan MP, Sherman ME. Etiology of hormone receptor-defined breast cancer: a systematic review of the literature. Cancer Epidemiol Biomarkers Prev. 2004; 13:1558-68. [PubMed: 15466970]

8. Anderson WF, Jatoi I, Devesa SS. Distinct breast cancer incidence and prognostic patterns in the NCI's SEER program: suggesting a possible link between etiology and outcome. Breast Cancer Res Treat. 2005; 90:127-37. [PubMed: 15803359]

9. Yang XR, Chang-Claude J, Goode EL, et al. Associations of breast cancer risk factors with tumor subtypes: a pooled analysis from the Breast Cancer Association Consortium studies. J Natl Cancer Inst. 2011; 103:250-63. [PubMed: 21191117]

10. Cotterchio M, Kreiger N, Theis B, Sloan M, Bahl S. Hormonal factors and the risk of breast cancer according to estrogen- and progesterone-receptor subgroup. Cancer Epidemiol Biomarkers Prev. 2003; 12:1053-60. [PubMed: 14578142]

11. Enger SM, Ross RK, Paganini-Hill A, Carpenter CL, Bernstein L. Body size, physical activity, and breast cancer hormone receptor status: results from two case-control studies. Cancer Epidemiol Biomarkers Prev. 2000; 9:681-7. [PubMed: 10919738]

12. Feigelson HS, Patel AV, Teras LR, Gansler T, Thun MJ, Calle EE. Adult weight gain and histopathologic characteristics of breast cancer among postmenopausal women. Cancer. 2006; 107:12-21. [PubMed: 16718671]

13. Huang WY, Newman B, Millikan RC, Schell MJ, Hulka BS, Moorman PG. Hormone-related factors and risk of breast cancer in relation to estrogen receptor and progesterone receptor status. Am J Epidemiol. 2000; 151:703-14. [PubMed: 10752798]

14. Li CI, Malone KE, Daling JR. Interactions between body mass index and hormone therapy and postmenopausal breast cancer risk (United States). Cancer Causes Control. 2006; 17:695-703. [PubMed: 16633917]

15. Rosenberg LU, Einarsdottir K, Friman EI, et al. Risk factors for hormone receptor-defined breast cancer in postmenopausal women. Cancer Epidemiol Biomarkers Prev. 2006; 15:2482-8. [PubMed: 17164374]

16. Setiawan VW, Monroe KR, Wilkens LR, Kolonel LN, Pike MC, Henderson BE. Breast cancer risk factors defined by estrogen and progesterone receptor status: the multiethnic cohort study. Am J Epidemiol. 2009; 169:1251-9. [PubMed: 19318616]

17. Suzuki R, Rylander-Rudqvist T, Ye W, Saji S, Wolk A. Body weight and postmenopausal breast cancer risk defined by estrogen and progesterone receptor status among Swedish women: A prospective cohort study. Int J Cancer. 2006; 119:1683-9. [PubMed: 16646051]

18. Wenten M, Gilliland FD, Baumgartner K, Samet JM. Associations of weight, weight change, and body mass with breast cancer risk in Hispanic and non-Hispanic white women. Ann Epidemiol. 2002; 12:435-4. [PubMed: 12160603]

19. Berstad P, Coates RJ, Bernstein L, et al. A case-control study of body mass index and breast cancer risk in white and African-American women. Cancer Epidemiol Biomarkers Prev. 2010; 19:153244. [PubMed: 20501755]

20. Lahmann PH, Hoffmann K, Allen N, et al. Body size and breast cancer risk: findings from the European Prospective Investigation into Cancer And Nutrition (EPIC). Int J Cancer. 2004; 111:762-71. [PubMed: 15252848] 
21. Morimoto LM, White E, Chen Z, et al. Obesity, body size, and risk of postmenopausal breast cancer: the Women's Health Initiative (United States). Cancer Causes Control. 2002; 13:741-51. [PubMed: 12420953]

22. Trentham-Dietz A, Newcomb PA, Egan KM, et al. Weight change and risk of postmenopausal breast cancer (United States). Cancer Causes Control. 2000; 11:533-42. [PubMed: 10880035]

23. Meijer K, de Vries M, Al-Lahham S, et al. Human primary adipocytes exhibit immune cell function: adipocytes prime inflammation independent of macrophages. PLoS One. 2011; 6:e17154. [PubMed: 21448265]

24. Rodriguez-Acebes S, Palacios N, Botella-Carretero J, et al. Gene expression profiling of subcutaneous adipose tissue in morbid obesity using a focused micrarray: Distinct expression of cell-cycle-and differentiation-related genes. BMC Medical Genomics. 2010; 3:1-15. [PubMed: 20092628]

25. Srdic B, Stokic E, Korac A, Ukropina M, Velickovic K, Breberina M. Morphological characteristics of abdominal adipose tissue in normal-weight and obese women of different metabolic profiles. Exp Clin Endocrinol Diabetes. 2010; 118:713-8. [PubMed: 20533176]

26. Villa J, Pratley RE. Adipose tissue dysfunction in polycystic ovary syndrome. Curr Diab Rep. 2011; 11:179-84. [PubMed: 21424395]

27. Friedenreich CM, Courneya KS, Bryant HE. Case-control study of anthropometric measures and breast cancer risk. Int J Cancer. 2002; 99:445-52. [PubMed: 11992416]

28. Huang Z, Willett WC, Colditz GA, et al. Waist circumference, waist:hip ratio, and risk of breast cancer in the Nurses' Health Study. Am J Epidemiol. 1999; 150:1316-24. [PubMed: 10604774]

29. Bernstein L, Allen M, Anton-Culver H, et al. High breast cancer incidence rates among California teachers: results from the California Teachers Study (United States). Cancer Causes Control. 2002; 13:625-35. [PubMed: 12296510]

30. Molarius A, Seidell JC. Selection of anthropometric indicators for classification of abdominal fatness--a critical review. Int J Obes Relat Metab Disord. 1998; 22:719-27. [PubMed: 9725630]

31. Horn-Ross PL, Lee VS, Collins CN, et al. Dietary assessment in the California Teachers Study: reproducibility and validity. Cancer Causes Control. 2008; 19:595-603. [PubMed: 18256894]

32. Kushi LH, Kaye SA, Folsom AR, Soler JT, Prineas RJ. Accuracy and reliability of selfmeasurement of body girths. Am J Epidemiol. 1988; 128:740-8. [PubMed: 3421240]

33. Hofer, BM.; Kwong, SL.; Allen, M.; Bates, JM.; Snipes, KP. Cancer in California, 1988-2007. California Department of Public Health, Cancer Surveillance Section; Sacramento, CA: 2010.

34. Allison, PD. Survival Analysis Using SAS: A Practical Guide. SAS Institute Inc; Cary, NC: 1995.

35. Potter JD, Cerhan JR, Sellers TA, et al. Progesterone and estrogen receptors and mammary neoplasia in the Iowa Women's Health Study: how many kinds of breast cancer are there? Cancer Epidemiol Biomarkers Prev. 1995; 4:319-26. [PubMed: 7655325]

36. Palmer JR, Adams-Campbell LL, Boggs DA, Wise LA, Rosenberg L. A prospective study of body size and breast cancer in black women. Cancer Epidemiol Biomarkers Prev. 2007; 16:1795-802. [PubMed: 17855697]

37. Suzuki R, Orsini N, Saji S, Key TJ, Wolk A. Body weight and incidence of breast cancer defined by estrogen and progesterone receptor status--a meta-analysis. Int J Cancer. 2009; 124:698-712. [PubMed: 18988226]

38. Vrieling A, Buck K, Kaaks R, Chang-Claude J. Adult weight gain in relation to breast cancer risk by estrogen and progesterone receptor status: a meta-analysis. Breast Cancer Res Treat. 2010; 123:641-9. [PubMed: 20711809]

39. Colditz GA, Rosner B. Cumulative risk of breast cancer to age 70 years according to risk factor status: data from the Nurses' Health Study. Am J Epidemiol. 2000; 152:950-64. [PubMed: 11092437]

40. Green J, Cairns BJ, Casabonne D, et al. Height and cancer incidence in the Million Women Study: prospective cohort, and meta-analysis of prospective studies of height and total cancer risk. Lancet Oncol. 2011; 12:785-94. [PubMed: 21782509]

41. Bao PP, Shu XO, Gao YT, et al. Association of hormone-related characteristics and breast cancer risk by estrogen receptor/progesterone receptor status in the shanghai breast cancer study. Am J Epidemiol. 2011; 174:661-71. [PubMed: 21768404] 
42. Pinheiro RL, Sarian LO, Pinto-Neto AM, Morais S, Costa-Paiva L. Waist circumference and waist to hip ratio do not contribute additional information on hormone receptor status of breast tumors in obese women. Breast J. 2010; 16:323-4. [PubMed: 20408825]

43. Ma H, Wang Y, Sullivan-Halley J, et al. Breast cancer receptor status: do results from a centralized pathology laboratory agree with SEER registry reports? Cancer Epidemiol Biomarkers Prev. 2009; 18:2214-20. [PubMed: 19661080]

44. Marshall SF, Clarke CA, Deapen D, et al. Recent breast cancer incidence trends according to hormone therapy use: the California Teachers Study. Breast Cancer Res. 2010; 12:R4. [PubMed: 20064209] 


\section{Table 1}

Selected characteristics of the California Teachers Study participants included in the present analysis $(\mathrm{N}=\underline{52,642})$

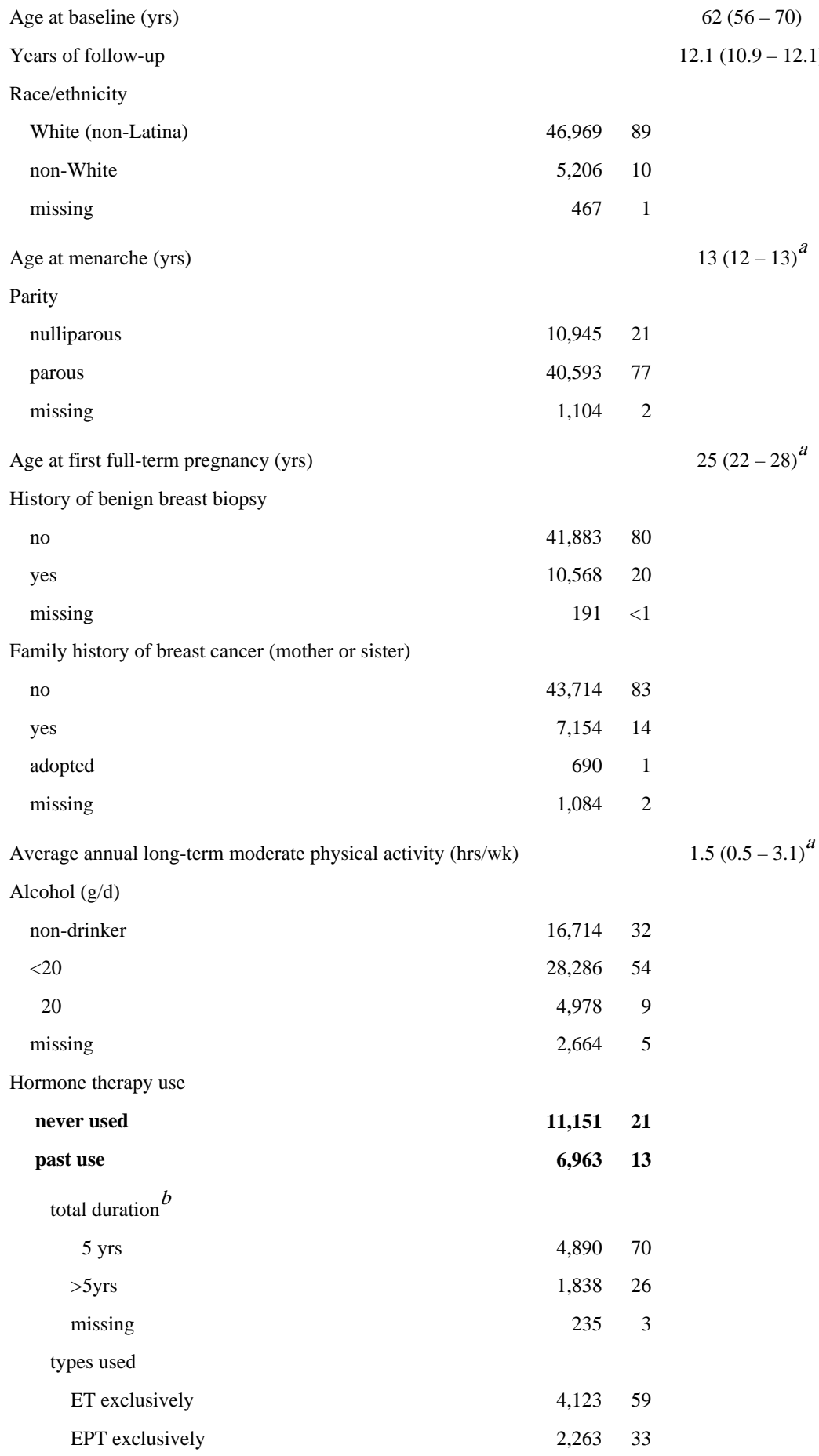




ET and EPT
using ET
total duration ${ }^{b}$
5yrs
$>5$ yrs
missing
types used
ET exclusively
past EPT use
using EPT
total duration ${ }^{b}$
5 yrs
$>5$ yrs
missing
types used
EPT exclusively
past ET use
missing
Height (in)
$<61$
$61-62$
$63-64$
$65-66$
$67-68$
$\times 69$

Body mass index $\left(\mathrm{kg} / \mathrm{m}^{2}\right)$

$<20$

20-24

25-29

30-39

$\geq 40$

Body mass index $\left(\mathrm{kg} / \mathrm{m}^{2}\right)$ at age 18 $<20$

20-24

25-29

$\geq 30$

missing

Weight change since age 18 loss $\geq 20$ pounds 10-19 pounds stable

\begin{tabular}{rr}
$\mathbf{N}$ & $\mathbf{\%}$ \\
\hline 577 & 8 \\
$\mathbf{1 4 , 4 4 7}$ & $\mathbf{2 7}$ \\
& \\
2,782 & 19 \\
11,049 & 77 \\
616 & 4
\end{tabular}

$11,405 \quad 79$

$3,042 \quad 21$

$15,005 \quad 29$

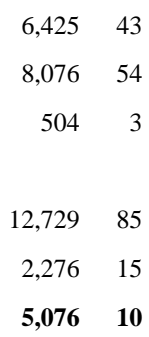


gain 10-24 pounds

25-39 pounds

40-49 pounds

250 pounds

missing

Waist circumference (in) ${ }^{c}$

$<30$

30-32

33-35

36-38

39-41

$\geq 42$

Waist-to-height ratio ${ }^{c}$

$<0.45$

0.45-0.49

$0.50-0.55$

$\searrow 0.56$

$I Q R$ interquartile range, $E T$ estrogen-only therapy, $E P T$ combined estrogen plus progesterone therapy

${ }^{a}$ Data missing for $\_\%$ of participants.

$b_{\text {Includes use of ET and EPT. }}$

${ }^{c}$ Available only for participants who completed the 1997-98 follow-up questionnaire.

\begin{tabular}{|c|c|c|}
\hline $\mathbf{N}$ & $\%$ & median (IQR) \\
\hline 13,495 & 26 & \\
\hline 9,728 & 18 & \\
\hline 4,566 & 9 & \\
\hline 8,473 & 16 & \\
\hline \multirow[t]{2}{*}{749} & 1 & \\
\hline & & $33(30-36)$ \\
\hline 8,529 & 24 & \\
\hline 9,005 & 25 & \\
\hline 7,401 & 21 & \\
\hline 4,997 & 14 & \\
\hline 2,878 & 8 & \\
\hline \multirow[t]{2}{*}{2,719} & 8 & \\
\hline & & $0.50(0.45-0.56)$ \\
\hline 6,922 & 19 & \\
\hline 9,647 & 27 & \\
\hline 9,200 & 26 & \\
\hline 9,760 & 27 & \\
\hline
\end{tabular}




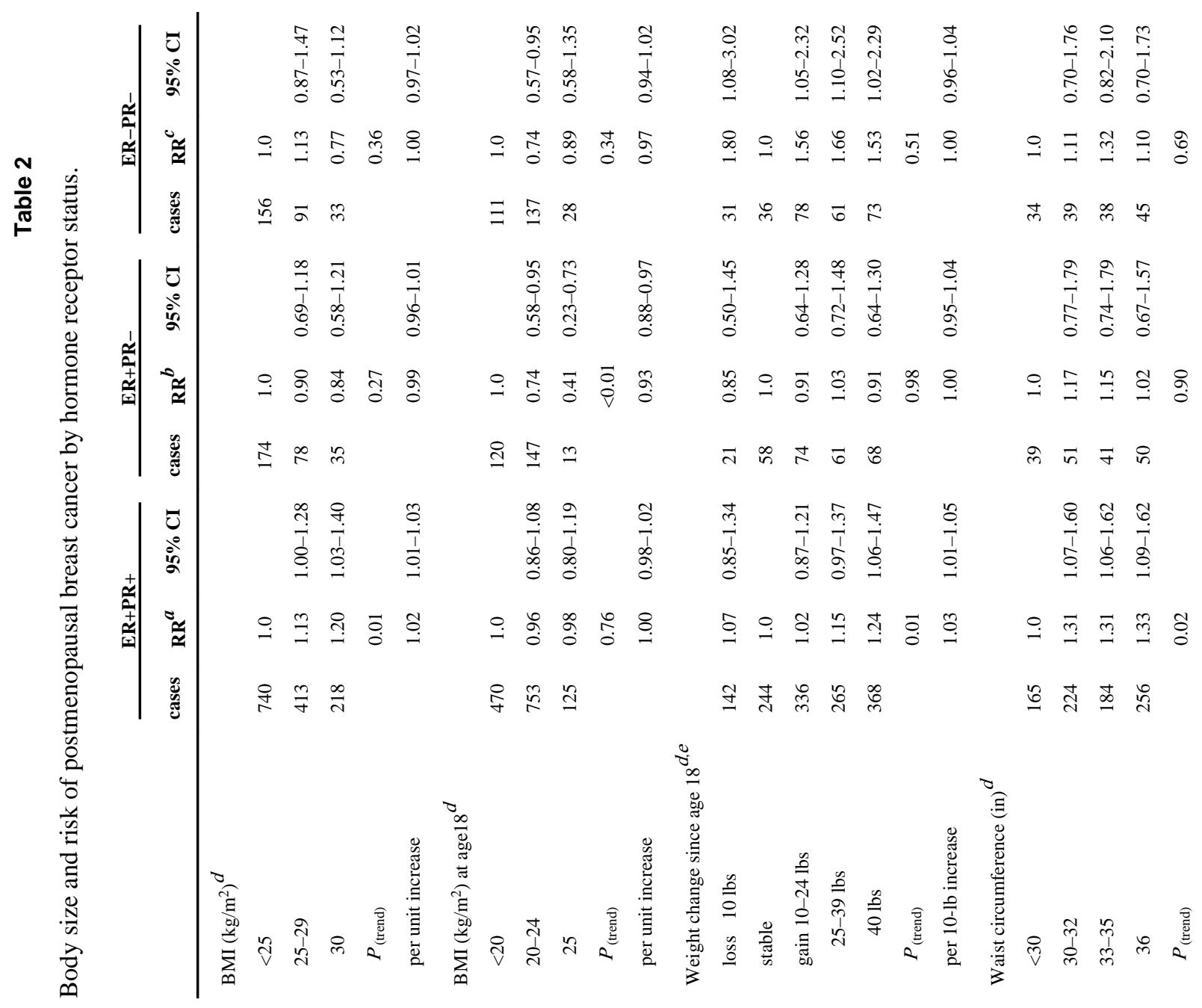




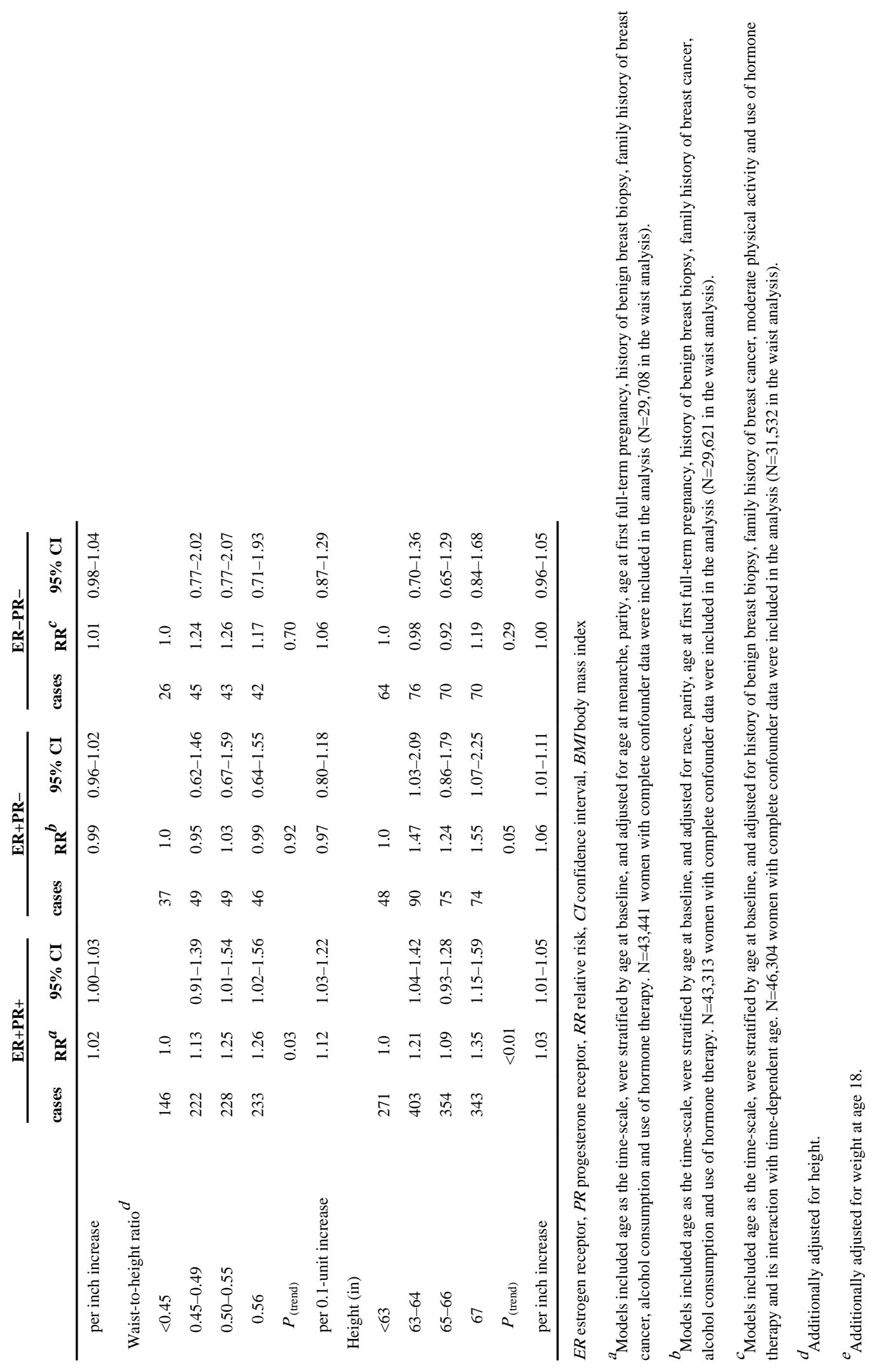



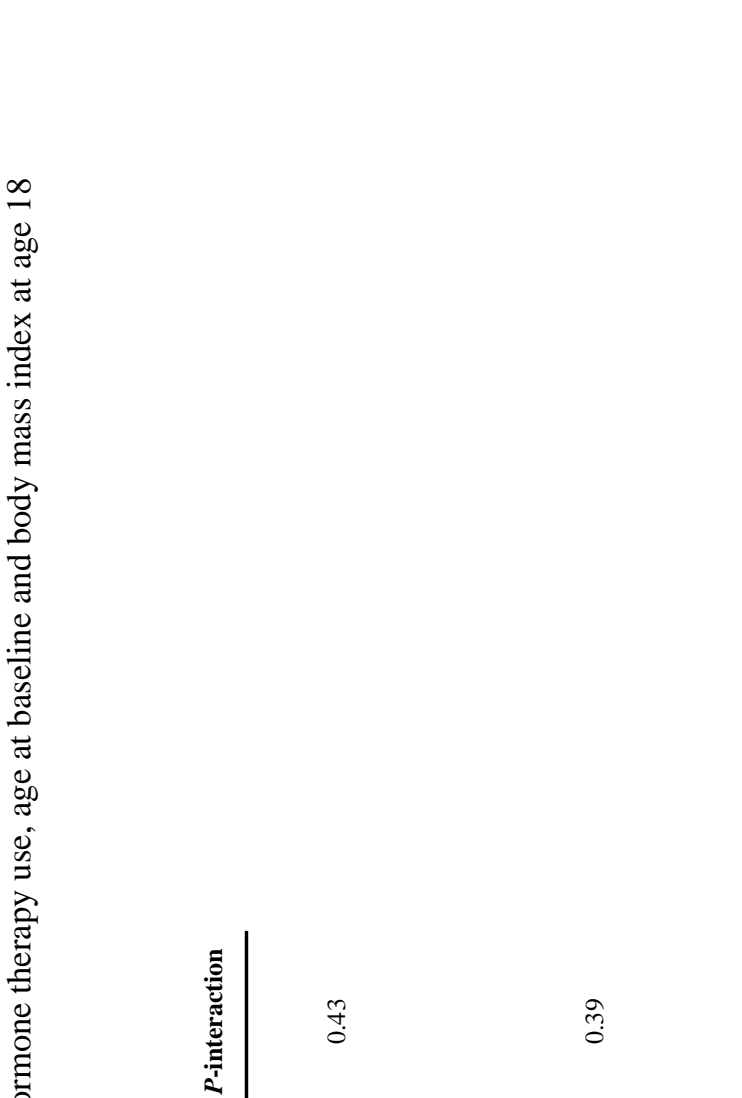

궁

$\stackrel{9}{8}$

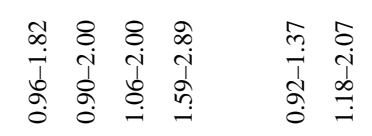

ते शे के

กี थै ๆ

- กิ

政

暠

高

总

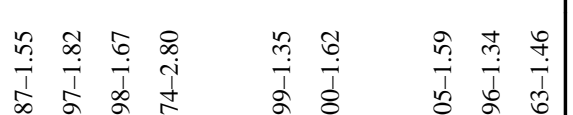
在

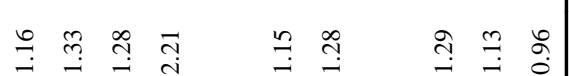

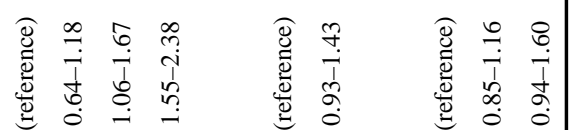

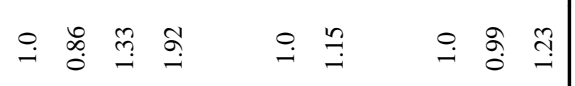
$\circ$

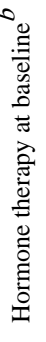

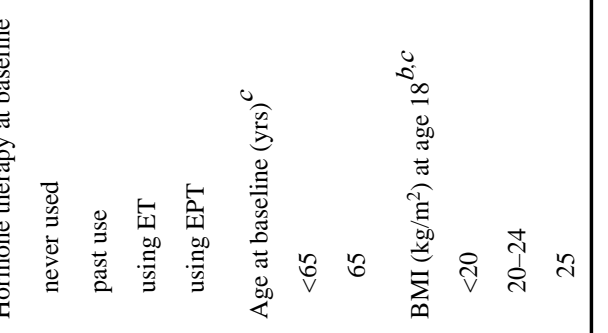




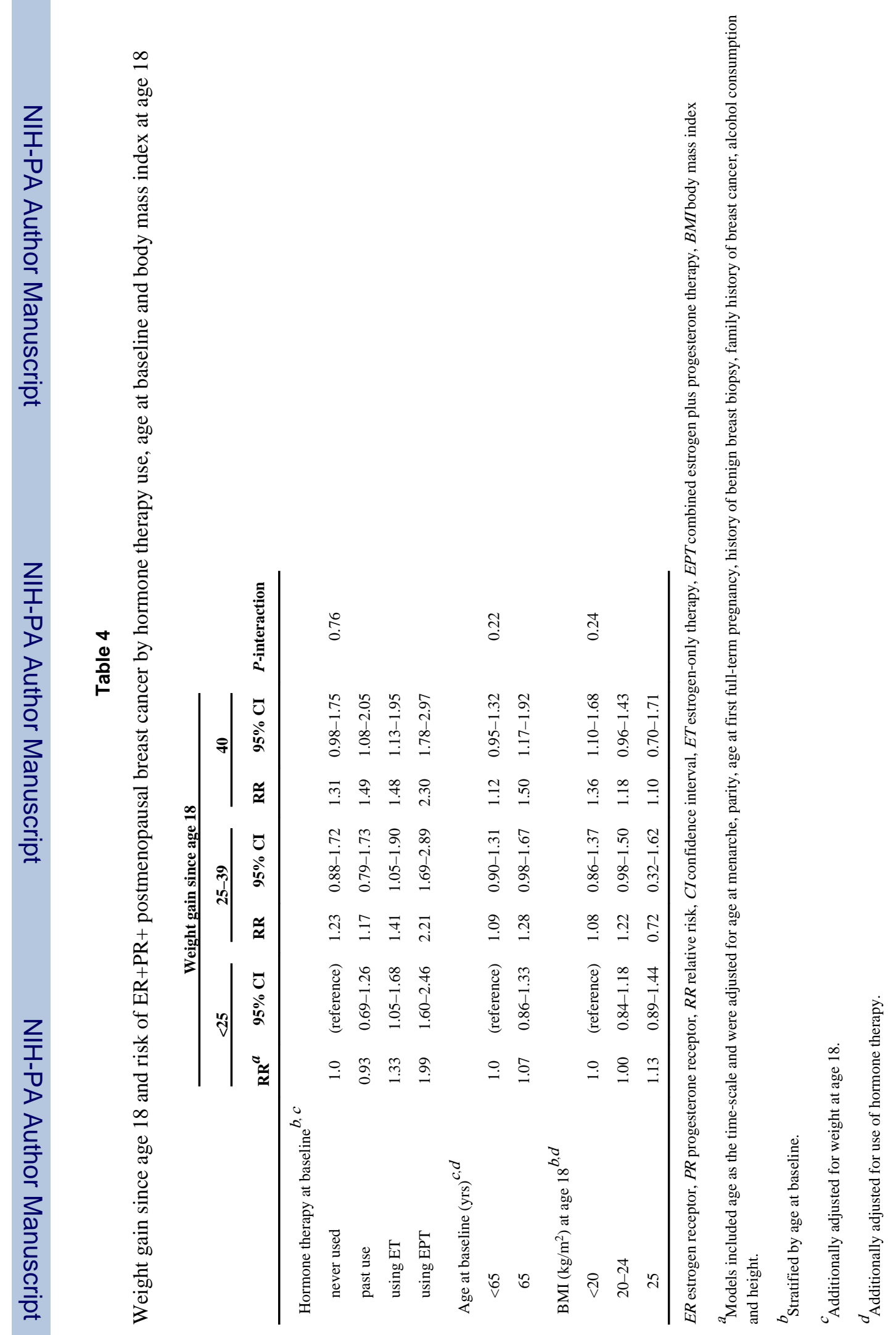




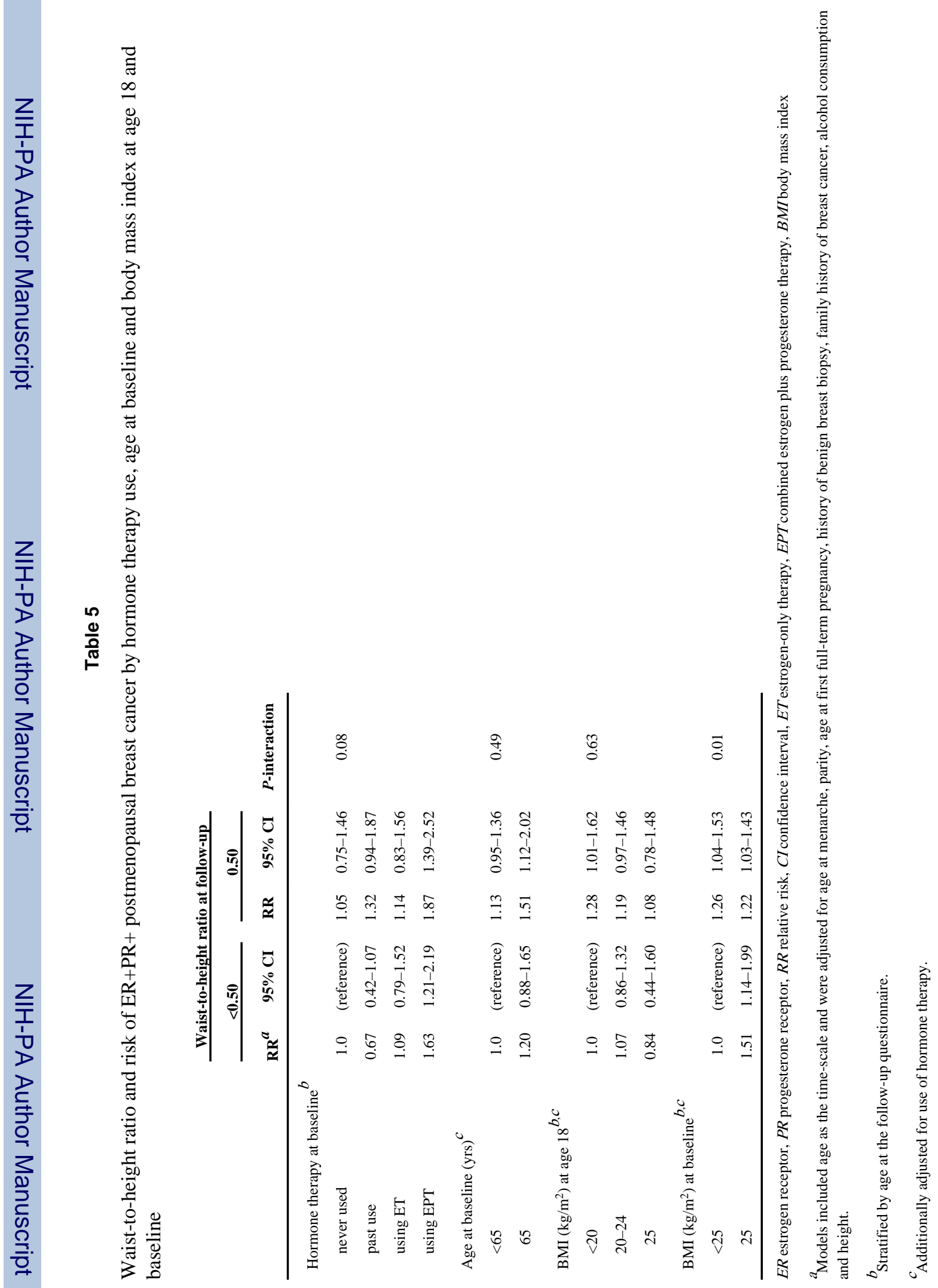

